\title{
An evaluation of the design of room characteristics of a sample of healing gardens
}

\author{
K. Maikov, S. Bell \& K. Sepp \\ Landscape Architecture Department, \\ Estonian University of Life Sciences, Estonia
}

\begin{abstract}
The present paper discusses the evaluation and description of the landscape characteristics of a sample of healing gardens from the USA and England. Healing gardens are designed in such a way as to influence the visitor by provoking positive emotions and to help relieve the symptoms of stress or depression. The aim of the paper is to analyse a sample of healing gardens on the basis of eight characteristics that, according to the literature, should be present in a garden for it to be classified as a healing garden and that may also be considered some of the fundamental building blocks of parks and gardens in general. The term "room" is used to convey a sense that each garden consists of a set of distinct spaces that are separated from each other and which are experienced by visitors as part of a sequence. Rooms can be generally described using a specific name to which a set of characteristics is attached. Each room type (Serene, Wild, Rich in Species, Space, the Common, the Pleasure garden, Festive, Culture) was evaluated in terms of the degree to which the characteristics were present using the following scale: 0 - not present, 1-weak, 2 - medium, 3 - strong. Following this a description of the design characteristics was carried out from the point of view of landscape design elements and structure, including an assessment of presence of the sense of the personal/impersonal. The purpose of the investigation is to determine which characteristics are mostly frequently used in the design of healing gardens, which characteristics most strongly feature and to analyse the main design elements. In total, 40 healing gardens were visited, 20 from England (summer 2005) and 20 from various states in New England in the USA (summer 2006). There were no apparent differences between the characteristics of healing garden rooms between the countries (t-test). Correlations were found between the types "Rich in Species" and "Festive" $(0.85)$ and "Culture" $(0.85)$ which tends to be found together in the gardens. The types "Space" and "The Common" had a high correlation (0.8) in England. "Rich in Species" and "Festive" had a correlation (0.9) in the USA. Of the gardens studied, the types "Rich in Species" and "the Common" are distinguished by the strongest presence. The personal characteristic was found to be present less than the impersonal. The characteristic "The Pleasure garden" does not exist in most of the gardens - it is possibly an expensive solution. The existence of the characteristics in the gardens does not depend on the idea of the design as a whole, and the strength of the characteristics will tend to influence the users the most.
\end{abstract}

Keywords: healing garden, design, room characteristics, USA, England. 


\section{Introduction}

Berggren-Bärring and Grahn have evolved the concept of the landscape room containing different characteristics [1] that are necessary for a landscape in order to make it a healing landscape [8]. The idea of a room is a separate space that may be experienced separately from other spaces, perhaps in a predefined sequence, in a garden or landscape. The characteristics of each room type together constitute symbols that manifest themselves through many different sensations: via sight, hearing, locomotion, etc [3-5, 8].

Some landscape room characteristics are found in conventional gardens, but their presence alone does not make a garden a healing garden (in this context to help the recovery from a number of mental health problems/illnesses such as clinical depression). A healing garden is therefore designed specifically to provide experiences to users through their senses, speeds up the healing process involving other therapies and study methods and gives support for low-key actions. The environment of a healing garden is bounded/guided/involving.

The experience of nature affects people differently, depending in part on their life situation [11]. There are different levels of action by human beings in outdoor areas (ranging from active behaviour with other people to sitting alone). How much does the environment support these actions? People use their senses differently on different occasions, but if outdoor situations (wind, sun, colours) are normal, there is participation in the environment by the senses on two different levels:

a) Impersonal experience: looking, hearing, smelling - receiving stimuli passively

b) Personal experience: looking, hearing, smelling + touching and tasting seeking out stimuli actively.

In everyday life, people may tend to use the first level and, for deeper purposes, the second level. Among the eight selected characteristics studied in this research, five of them can be considered as impersonal room characteristics (see Appendix 1). In addition, the environment itself will suggest possible actions to the visitor. For healing purposes, the garden user has to engage with the landscape at the personal level.

Nowadays, in landscape design it may not be enough to deal merely with classical design themes, styles or client's wishes, but also its healing or restorative aspects should be given more attention. Design therefore should provide more than a sequence of outdoor spaces or rooms and views, but also develop place identity and if desired, should support different healing stages (by the use of stone/water, plant, animals, other people). There has been a little evaluation of the garden rooms where the healing process takes place outdoors. Area range of different therapies may be used by practitioners in the garden, but how is it possible to tell if the garden supports action? What characteristics occur most frequently in the rooms? Which of them are used less and why? If only impersonal and personal possibilities are taken into account without considering what people can do in different areas possessing different characteristics - there are interesting values which may remain hidden. 
The healing gardens assessed in this study are generally not publicly accessible and are mainly used by patients and staff in different hospitals or institutions. In England the gardens are, as a rule, meant for purposes of rehabilitation or general use by convalescing people in hospitals, while in the United States they are for general use in hospitals. The gardens of both countries belong to two different generations of design and degree of maturity. In most gardens, cognitive therapies are used by therapists with different patients.

\section{Method}

The sample of healing gardens was obtained from a public database [6] and the compliance of these with best practice was checked from published literature. Altogether, 40 healing gardens were visited, 20 in England (summer 2005) and 20 from different states in New England in the USA (summer 2006). The choice was based only on the presence of a garden region. Garden types (such as specifically for Alzheimer patients, rehabilitation, child treatment, etc.) were not differentiated in this study because the sample size of each was insufficient for statistical analysis. Where possible, the healing gardens were designed to be divided into physical "rooms" - some being more natural areas, made of living materials and bordered with trees as enclosure and separation from each other. Each "room" was evaluated separately.

Each room was evaluated in terms of the degree of presence of the thematic attribute: "Serene", "Wild", "Rich in Species", "Space", The Common", "The Pleasure Garden", "Festive" and "Culture" (see Table 1) using a four-point scale ( 0 - not present, 1 - weak presence, 2 - medium presence, 3 - strong presence). Table 2 describes the criteria for each theme against the scale. There were no statistically significant differences between the characteristics of healing garden rooms in either country (t-test).

\section{Results}

Table 3 shows the complete set of scores for each room type across the samples, firstly for the entire sample and then the English sample followed by the American sample. Bold numbers denote scores with a significance of density of appearance.

\subsection{Analysis of the characteristics of the sampled healing gardens}

Overall, a high proportion of the evaluated characteristics received a very low score. The quality of the gardens and characteristics showed extreme values: there were some gardens, the design of which included all aspects while others only included a few characteristics. The most strongly represented characteristic in most gardens is "Serene" (55\% of the cases) while the "Pleasure garden" is rarely found $(25 \%$ of the cases). Other characteristics tend to be show a weak presence $(40 \%)$. 
Table 1: Name and description of the room characteristics used in the study [1] with personal-impersonal division and picture by author.

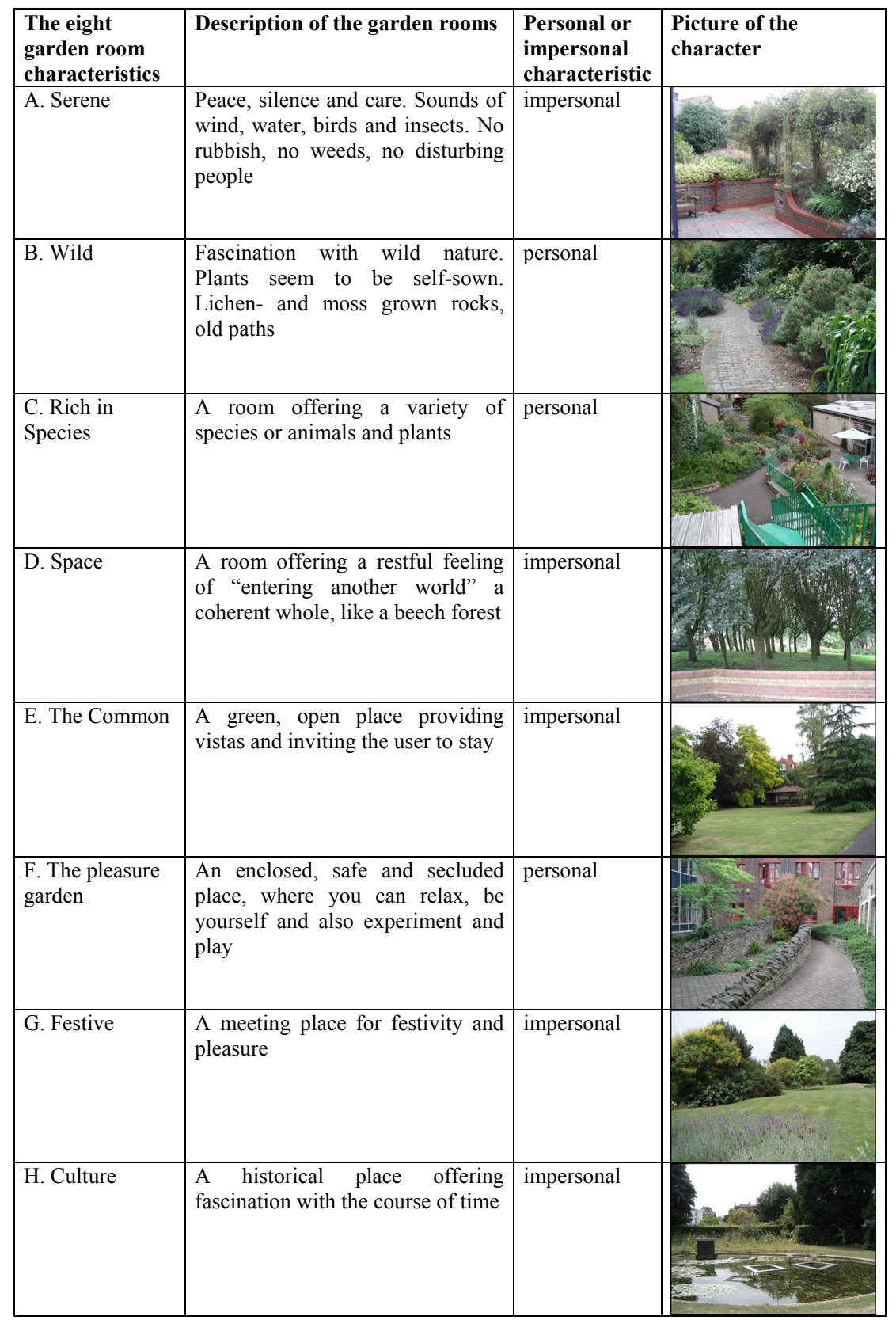


Table 2: $\quad$ Description of ratings.

Serene

\begin{tabular}{|l|l|}
\hline 1 & None \\
\hline 2 & Too much confusion to the senses - does not achieve the goal \\
\hline 3 & Is present and produces the desired sensations \\
\hline 4 & $\begin{array}{l}\text { All characteristics are present in all rooms. Strong man-made feeling yet powerful sense of } \\
\text { the serene }\end{array}$ \\
\hline
\end{tabular}

\begin{tabular}{|l|l|} 
Wild \\
\hline 1 & None \\
\hline 2 & $\begin{array}{l}\text { Link to nature with at least one characteristic (e.g. Wild trees). Weakly tied with "wild" } \\
\text { character meaning. }\end{array}$ \\
\hline 3 & Connects to nature. Living material naturally belongs there. \\
\hline 4 & Deep fascination of nature with culture taste \\
\hline
\end{tabular}

\section{Rich in Species}

\begin{tabular}{|l|l|}
\hline 1 & None \\
\hline 2 & Not so rich in species. Design is developed more to reflect other characteristics \\
\hline 3 & $\begin{array}{l}\text { Different feelings in different rooms - made by variety of living material. On average rich } \\
\text { in species }\end{array}$ \\
\hline 4 & Rich in diversity of both animals and plants \\
\hline
\end{tabular}

\begin{tabular}{|l|l|}
\hline Space & \multicolumn{2}{|l|}{} \\
\hline 1 & None \\
\hline 2 & Space present, but no restful feeling \\
\hline 3 & Different characters in different rooms with strong ability to connect (eg beech, water) \\
\hline 4 & To "other world" through use of plants \\
\hline
\end{tabular}

\section{The Common}

\begin{tabular}{|l|l|}
\hline 1 & None \\
\hline 2 & $\begin{array}{l}\text { Present in lonely places/corners where some interesting design solutions are used. Consists } \\
\text { of only one element }\end{array}$ \\
\hline 3 & $\begin{array}{l}\text { Green, open, but does not invite the user to sit there or invites the user to sit, but is not } \\
\text { open: incomplete character }\end{array}$ \\
\hline 4 & All elements are present, \\
\hline
\end{tabular}

\section{The Pleasure garden}

\begin{tabular}{|l|l|}
\hline 1 & None \\
\hline 2 & Weak, pleasurable aspects are not well designed together \\
\hline 3 & Close, safe, separated, user can be his or herself in a well designed setting \\
\hline 4 & All features present, one room available for one person to enjoy \\
\hline
\end{tabular}

\section{Festive}

\begin{tabular}{|l|l|}
\hline 1 & None \\
\hline 2 & Presented weakly, with only one key character present \\
\hline 3 & Both characters present but, one stronger than the other or neither very strong \\
\hline 4 & Easy, open space offering gatherings in any way ; versatile space \\
\hline
\end{tabular}

\section{Culture}

\begin{tabular}{|l|l|}
\hline 1 & None \\
\hline 2 & Presented weakly, attractive for a short time, user can enjoy touching an object \\
\hline 3 & $\begin{array}{l}\text { One main element present enabling the user to forget the time - connecting the users of the } \\
\text { area }\end{array}$ \\
\hline 4 & $\begin{array}{l}\text { Significant part of the garden, strong characteristics presented enabling users to forget the } \\
\text { time and environment completely }\end{array}$ \\
\hline
\end{tabular}


Table 3: $\quad$ Evaluation results of marks together and by country in graphic.

All together

\begin{tabular}{|r|r|r|r|r|r|r|r|r|}
\hline & $\mathbf{A}$ & $\mathbf{B}$ & $\mathbf{C}$ & $\mathbf{D}$ & $\mathbf{E}$ & $\mathbf{F}$ & G & H \\
\hline $\mathbf{3}$ & $\mathbf{5 5 , 0} \%$ & $15,0 \%$ & $22,5 \%$ & $17,5 \%$ & $22,5 \%$ & $15,0 \%$ & $7,5 \%$ & $10,0 \%$ \\
\hline $\mathbf{2}$ & $17,5 \%$ & $15,0 \%$ & $22,5 \%$ & $20,0 \%$ & $17,5 \%$ & $7,5 \%$ & $\mathbf{2 5 , 0} \%$ & $17,5 \%$ \\
\hline $\mathbf{1}$ & $17,5 \%$ & $\mathbf{3 7 , 5} \%$ & $\mathbf{4 0 , 0} \%$ & $\mathbf{3 7 , 5} \%$ & $\mathbf{4 5 , 0} \%$ & $2,5 \%$ & $\mathbf{4 7 , 5 \%}$ & $\mathbf{4 0 , 0} \%$ \\
\hline $\mathbf{0}$ & $10,0 \%$ & $\mathbf{3 2 , 5} \%$ & $15,0 \%$ & $\mathbf{2 5 , 0} \%$ & $15,0 \%$ & $\mathbf{7 5 , 0} \%$ & $20,0 \%$ & $32,5 \%$ \\
\hline
\end{tabular}

England

\begin{tabular}{|r|r|r|r|r|r|r|r|r|}
\hline & A & B & C & D & E & F & G & H \\
\hline $\mathbf{3}$ & $\mathbf{5 5 , 0} \%$ & $\mathbf{2 5 , 0} \%$ & $\mathbf{3 0 , 0} \%$ & $30,0 \%$ & $20,0 \%$ & $10,0 \%$ & $10,0 \%$ & $15,0 \%$ \\
\hline $\mathbf{2}$ & $15,0 \%$ & $5,0 \%$ & $15,0 \%$ & $5,0 \%$ & $20,0 \%$ & $10,0 \%$ & $20,0 \%$ & $5,0 \%$ \\
\hline $\mathbf{1}$ & $20,0 \%$ & $\mathbf{3 5 , 0} \%$ & $\mathbf{4 0 , 0} \%$ & $\mathbf{4 0 , 0} \%$ & $\mathbf{5 0 , 0} \%$ & $5,0 \%$ & $\mathbf{4 5 , 0} \%$ & $\mathbf{4 0 , 0} \%$ \\
\hline $\mathbf{0}$ & $10,0 \%$ & $\mathbf{3 5 , 0} \%$ & $15,0 \%$ & $\mathbf{2 5 , 0} \%$ & $10,0 \%$ & $\mathbf{7 5 , 0} \%$ & $\mathbf{2 5 , 0} \%$ & $\mathbf{4 0 , 0} \%$ \\
\hline
\end{tabular}

USA

\begin{tabular}{|r|r|r|r|r|r|r|r|r|}
\hline & \multicolumn{1}{|l|}{} & $\mathbf{B}$ & $\mathbf{C}$ & $\mathbf{D}$ & $\mathbf{E}$ & F & G & H \\
\hline $\mathbf{3}$ & $\mathbf{5 5 , 0} \%$ & $5,0 \%$ & $15,0 \%$ & $5,0 \%$ & $\mathbf{2 5 , 0} \%$ & $20,0 \%$ & $5,0 \%$ & $5,0 \%$ \\
\hline $\mathbf{2}$ & $20,0 \%$ & $\mathbf{2 5 , 0} \%$ & $\mathbf{3 0 , 0} \%$ & $\mathbf{3 5 , 0} \%$ & $15,0 \%$ & $5,0 \%$ & $\mathbf{3 0 , 0} \%$ & $\mathbf{3 0 , 0} \%$ \\
\hline $\mathbf{1}$ & $15,0 \%$ & $\mathbf{4 0 , 0} \%$ & $\mathbf{4 0 , 0} \%$ & $\mathbf{3 5 , 0} \%$ & $\mathbf{4 0 , 0} \%$ & $0,0 \%$ & $\mathbf{5 0 , 0} \%$ & $\mathbf{4 0 , 0} \%$ \\
\hline $\mathbf{0}$ & $10,0 \%$ & $\mathbf{3 0 , 0} \%$ & $15,0 \%$ & $\mathbf{2 5 , 0} \%$ & $20,0 \%$ & $\mathbf{7 5 , 0} \%$ & $15,0 \%$ & $\mathbf{2 5 , 0} \%$ \\
\hline
\end{tabular}

In England, the healing gardens sampled are designed with strong constructed views, a sense of cohesion and use of natural voices, with non-disturbing factors used to induce calm. On the basis of the evaluation results, the strongest scores were: "Serene" - 55\%, "Rich in Species" - 30\% and "Wild" - 25\%. There is a tendency for country gardens to be more nature-oriented in their design and to have more opportunities for personal contact. The "Pleasure Garden" was only found in $25 \%$ of the cases. Other characteristics are found to a lower degree. The two themes "Space" and "The Common" were strongly correlated (0.8), indicating that they are frequently found together in gardens. This could be accounted for by the use of the typical approaches to English garden design.

In the USA, the same characteristics tended to have lower scores than in England. High scores tended to be associated with impersonal characteristics like "Serene" $-55 \%$ and "The Common" - 25\%. While other characteristics were almost equally represented at a low level in England, by contrast the gardens of the American sample show a low and middle level of presence (average of $40 \%$ ). Cohesive characteristics are associated strongly with "Festivity" and the "Pleasure Garden" - in both cases the correlation was (0.9). There are fewer personal characteristics to be found in the landscape than impersonal ones.

\subsection{Completion of descriptions of room characteristics}

Detailed descriptions of the characteristics were developed for the evaluation of the garden rooms in a similar fashion to those adopted for parks in urban areas. 
The goal was to complete a description specifically considering aspects of healing gardens. Through the perspective of a landscape designer the following descriptions were developed from the initial descriptions found in BerggrenBärring and Grahn [1].

A. Serene You will find peace, silence and a sense that the emotions of the visitor are reflected by the room itself. Sounds of wind, water, birds and insects are present while rubbish, weeds, or disturbing people are absent [1]. Clean and clear areas in man-made nature are highly preferred. Well-cared for area and natural sounds are the key words that describe this theme, together with a strong safe man-made feeling. This atmosphere suits the purpose of the garden and has a safe environment, especially appropriate in hospital situations. The design should create as natural an environment as possible; the most frequent activities are one-person, passive and impersonal.

B. Wild This theme shows a strong fascination with wild nature. Plants are designed to seem self-sown. Lichen- and moss-grown rocks and old paths reinforce this [1]. Nature itself is a very inspiring element in the design. The room characteristic is achieved by playing with a mix of live and non-living or dead materials to show a sense of safe mixed nature. The purpose of the characteristic is to stimulate the user to see and touch, to make the user discover their surroundings and to make them feel. This can be used by groups and individuals with personal contacts included in the opportunities.

C. Rich in Species The room includes a variety of species of animals and plants [1]. The characteristics will show the diversity of nature. It is used to draw the users' attention to different elements, compositions, and colours. Richness itself offers personal contact and a lot of social factors between users and their inner life. A range of design techniques are strongly recommended for this theme.

D. Space This theme presents a room offering a restful feeling of "entering another world", a coherent whole, for example a beech forest [1] or Salix fragilis 'Bullata'. There is a good opportunity to create a single, simple structure in order to create or stimulate clear, easy understandable, mystical feelings. It allows for working more with the user's inner life, where it is good to use the environment simply to breathe in and out, enabling a person to stay in the area for a longer time. It shows an impersonal characteristic.

E. The Common A green, open place allowing vistas and encouraging users to stay [1]. It is an open characteristic room with another room material. Simple sitting opportunities with different types of views, vistas, textures and colours play in the distance, creating safe man-made views. In this open space the visitor is visible to other users and therefore is less likely to be as relaxed as in some of the other room types.

F. The Pleasure Garden An enclosed, safe and secluded place where you can relax and be yourself and also experiment and play [1]. It is usual to have small gardens suitable for individuals inside larger ones with promising elements to use. The gardens should be designed with living materials that can be moved from one place to another - big-leafed plants that provide a sense of security are preferred - so as to be able to create different spaces and experiences. This type is intended mainly for personal actions. 
G. Festive A meeting place for festivity and pleasure [1]. There are opportunities to feel free and without constraints. There could also be a small garden element for performance, such as an extra room, for example a lawn area for gathering on any occasion. This theme offers impersonal communication.

H. Culture A historical place, offering fascination with the passing of time [1]. It is good to focus on man-made or natural elements of different ages, such as stones. Materials such as stones or plants which change their texture or colour according to different weather conditions are also preferred. It is a room of opencharacter with some strong vistas.

\section{Discussion}

The aim of the design is to create garden rooms that are not too abstract, unfamiliar, or challenging [5], but supportive of the intended actions in terms of healing needs. According to Grahn, when person is going to the garden, then he or she first touches a stone or some water, then plants and animals after which he or she expects to see another human being. Some characteristics can be found almost everywhere in outdoor spaces, but this does not mean that these rooms or themes are linked or are related to the user in the same way as in a healing garden context.

The room characteristics under comparison received some extreme values, from very high to very low or absent. In most cases, the sampled gardens scored quite low ratings. The American sample contained most of the selected room characteristics, usually having average scores. According to Berggren-Bärring and Grahn of the eight garden room characteristics the types "Serene", "Space" and "Culture" appeal to many people. "The Common" tends to appeal to vulnerable people and the "Festive" to stressed people. It is easy to understand the existence or non-existence of the characteristic in the landscape. However, the characteristics also have to be interpreted in terms of local meanings. The current study has also demonstrated that at least five of the eight characteristics should to be present in order for a garden to be classified as a healing garden, which confirmed the results of previous studies [7]. To see the situation in terms of personal/impersonal characteristics there should be more personal than impersonal. There could be more opportunities for landscape design to create more linkages between spatial elements, design materials, and people.

In terms of the analysis of the results some interesting pictures emerged. "Rich in Species" had high correlations with "Festive" (0.85) and "Culture" (0.85). This connection suggests that a good combination in a design includes all impersonal action categories are linked with personal contact possibilities through the richness of materials used to create a coherent design; the characteristics have to be more strongly differentiated in order to support the actions of users and to assure their safety. In the sample, the "Serene" and "Festive" are distinguished by a generally stronger presence.

There are also some differences between the gardens of England and the USA. English gardens are found to be more orientated towards closeness to nature than the American, which are more supportive of social actions. 
There are fewer personal characteristics found in the rooms than impersonal characteristics. It was also interesting that the "Pleasure garden" type is rarely found $(25 \%)$. The reason for this may be the expense of construction and maintenance. The types "Wild" and "Space" also received a low rating, 32.5\%, and $25 \%$ respectively.

There are two personal characteristics, "Wild" and "Rich in Species", that rated as being strong presences and which communicated the most with garden users; these are also the only room characteristics that are impossible to imitate indoors. It also appears that the characteristic "Festive", which helps to generate different feelings in different life situations and for the mood of users that it is most difficult characteristic for a designer to create.

The completion and extension of the characteristics of each theme type described here and related to user activities and therapeutic values should help designers to adapt and develop spaces so as to increase the healing potential of the rooms. This implies that following the descriptions requires understanding of the designer's point of view and language.

The evaluation method adopted in this study appeared to work well in differentiating between different room characteristics of all the samples and therefore it should be widely applicable as a tool for evaluating any healing garden.

The general idea was to find the key room characteristics that relate strongly to certain types of healing processes. Walking through the rooms helps users to orientate themselves and also to find the rhythm in the design. The next step of the scientific task is to find out if the order of the rooms is important for the most effective healing process. It is also important to understand how the message of the journey from room to room affects the healing process.

\section{Conclusions}

Evaluations of the characteristics of rooms within healing have so far not been attempted from the point of view of the design elements. This study has demonstrated that the eight room characteristics, originally developed for evaluation of urban parks, could also be used in the description and evaluation of healing gardens. The selected room characteristics are easy to recognise in healing gardens. This work has also added to the comprehension of the role of the personal and impersonal user opportunities and interprets them from the point of view of a landscape designer. Room characteristic descriptions were completed from the sample of gardens. The next stage of development is to understand the role of sequence and how to develop rooms that reflect different personal characteristics in order to relate to the user's inner life. The analysis of the strength of the existence of the characteristics yielded an extreme range in the English sample and a different set of associations in the American examples. "Rich in Species" had a high correlation with "Festive" $(0.85)$ and "Culture" (0.85). "Space" and "The Common" had a high correlation (0.8) in England. "Festivity" and the "Pleasure Garden" both showed a correlation of (0.9) in the USA. This work developed and completed descriptions of room characteristics 
so as to be useful for designers; the existence of these characteristics in the sample gardens showed some correlations. The current work provides a better guide to the design of healing gardens. The next steps, having improved the description of the individual room's types is to test the order of the rooms in different sequences to meet different therapeutic goals. It is also important to determine how such sequences contribute to the message of the journey and how this affects the therapeutic response.

\section{References}

[1] Berggren-Bärring A-M and Grahn P, Importance of the single park area on experienced characteristics. Ecological aspects of Green Areas in Urban Environments. IFPRA World Congress Proceeding, 110. Antwerp, Flanders, Belgium: September 1995

[2] Cooper Marcus and Barnes Healing gardens: Therapeutic benefits and design recommendations. New York: John Wiley and Sons 1999

[3] Grahn, P Om parkers betydelse. Stad \& Land. Movium/institutionen för landskapsplanering, Sveriges Lantbruksuniversitet, Alnarp. 1991

[4] Berggren-Bärring, A-M. \& Grahn, P. Grönstrukturens betydelse för användningen: En jämförande studie av hur människor i barnstugor, skolor, föreningar, vårdinstitutioner $\mathrm{m}$ fl organisationer utnyttjar tre städers parkutbud. Landskapsplanering Rapport 95:3, Alnarp. 1995

[5] Berggren-Bärring A-M. \& Grahn, P. Importance of the single park area on experienced characteristics. Ecological Aspects of Green Areas in Urban Environments. IFPRA World Congress Proceedings, 110. Antwerp, Flanders, Belgium: September. 1995

[6] Hedfors, P. \& Grahn, P. Soundscapes in urban and rural planning and design. Yearbook of Soundscape Studies 1: 67-82, 1998

[7] Maikov, K. A comparison of the room characteristics of healing gardens in landscape design on the example of the United States and England. COST E39 conference publications. 2007

[8] Stigsdotter U, Grahn P. What makes a garden a Healing garden? Journal of Therapeutic Horticulture, vol 13, pp 60-69, 2002

[9] Stigsdotter U, Grahn P. Experiencing a Garden: A Healing Garden for People Suffering from Burnout Diseases, Journal of Therapeutic Horticulture, vol 14, pp 38-49, 2003

[10] THERAPEUTIC LANDSCAPE DATABASE, http://www. healinglandscapes.org/, 12.06.2006

[11] Ottosson, J. \& Grahn, P. Utemiljöns betydelse för äldre med stort vårdbehov. Stad \& Land 155. Movium/instittutionen för landskapsplanering, Sveriges Lantbruksuniversitet, Alnarp 1998 